\title{
Household and Hospitalization Costs of Pediatric Dengue Illness in Colombo, Sri Lanka
}

\author{
Enoka Sonali Fernando, ${ }^{1}$ Tyler Y. Headley, ${ }^{2}$ Hasitha Tissera, ${ }^{3}$ Annelies Wilder-Smith, ${ }^{4,5,6}$ Amala De Silva, ${ }^{7}$ and Yesim Tozan ${ }^{2,8 *}$ \\ ${ }^{1}$ Health Office Egodauyana, Ministry of Health, Colombo, Sri Lanka; ${ }^{2}$ Department of Political Science, New York University Abu Dhabi, Abu \\ Dhabi, United Arab Emirates; ${ }^{3}$ Epidemiology Unit, Ministry of Health, Colombo, Sri Lanka; ${ }^{4}$ Heidelberg Institute of Global Health, University of \\ Heidelberg, Heidelberg, Germany; ${ }^{5}$ Department of Disease Control, London School of Hygiene and Tropical Medicine, London, United Kingdom; \\ ${ }^{6}$ Department of Epidemiology and Global Health, Umeå University, Umeå, Sweden; ${ }^{7}$ Department of Economics, University of Colombo, Colombo, Sri \\ Lanka; ${ }^{8}$ School of Global Public Health, New York University, New York, New York
}

\begin{abstract}
Dengue, a mosquito-borne viral infection that affects millions around the world, poses a substantial economic burden in endemic countries. We conducted a prospective costing study in hospitalized pediatric dengue patients at the Lady Ridgeway Hospital for Children (LRHC), a public pediatric hospital in Colombo district, Sri Lanka, to assess household out-of-pocket and hospitalization costs of dengue in pediatric patients during peak dengue transmission season. Between August and October 2013, we recruited 216 hospitalized patients (aged 0-3 years, 27\%; 4-7 years, 29\%; 8-12 years, 42\%) who were clinically or laboratory diagnosed with dengue. Using 2013 US dollars, household outof-pocket spending, on average, was US\$59 (SD 49) per episode and increased with disease severity (DF, US\$52; DHF/ DSS, US\$78). Pediatric dengue patients received free-of-charge medical care during hospitalization at LRHC, and this places a high financial burden on hospitals. The direct medical cost of hospitalization was US\$68 (SD 31.4) for DF episode, and US\$122.7 (SD 65.2) for DHF/DSS episode. Yet a hospitalized dengue illness episode still accounted for $20 \%$ to $35 \%$ of household monthly income due to direct and indirect costs. Additionally, a majority of caregivers $(70 \%)$ sought outpatient care before hospitalization, most of whom (81\%) visited private health facilities. Our findings indicate that hospitalized pediatric dengue illness poses a nontrivial cost burden to households and healthcare systems, emphasizing the importance of preventing and controlling the transmission of dengue in endemic countries.
\end{abstract}

\section{INTRODUCTION}

Dengue, a mosquito-borne viral disease infecting an estimated 390 million people annually, is increasing in prominence as a global health problem. ${ }^{1}$ Dengue has also become a leading cause of febrile illness in international travelers. ${ }^{2-6}$ Dengue infections may progress to dengue hemorrhagic fever (DHF) and dengue shock syndrome (DSS), which are associated with higher hospitalization and case-fatality rates, particularly among children. ${ }^{7-9}$ An estimated 40,500 (range: $17,600-49,800$ ) people die because of dengue-related complications worldwide annually. ${ }^{9}$ Dengue has received significant amount of research and development funding recently, but there are still critical unmet medical needs, such as specific antiviral drugs and vaccines. ${ }^{10}$ The intensification of dengue transmission, with frequent and severe epidemics, poses an enormous challenge to endemic countries with limited healthcare resources. Dengue surveillance systems in endemic countries rely on passive surveillance, which is a less resource-intensive approach, but it results in underreporting of cases. This hinders an accurate understanding of dengue's health and economic burden. ${ }^{11-13}$ Reliable country-specific burden estimates are sparse, ${ }^{14-17}$ and results are often not comparable due to a lack of uniformity in methods. ${ }^{18}$

Dengue is a leading public health priority in Sri Lanka. All four dengue serotypes cocirculate, and cases are reported from all districts. ${ }^{14,19,20}$ Over the past 15 years, progressively larger-scale epidemics have occurred at regular intervals. ${ }^{21}$ There have also been precipitous increases in dengue incidence and its severe manifestations. ${ }^{22,23}$ Both trends are associated with the spread of dengue viruses, changes in serotype distribution, ${ }^{24,25}$ and geographic expansion of $\mathrm{Ae}$ des vector mosquitoes ${ }^{19,26-28}$ and have occurred against a

*Address correspondence to Yesim Tozan, New York University School of Global Public Health, 14 East 4th Street, 3rd floor, New York, NY 10003. E-mail: tozan@nyu.edu backdrop of growing political commitment and funding to dengue control in Sri Lanka. ${ }^{29}$

Dengue can impose financial hardship on households in addition to its economic burden on healthcare systems in affected countries. Dengue illness leads to social and economic disruption at the household-level, requiring multiple visits to health care facilities, medical and non-medical expenditures, missed school and work days, and foregone household productivity. ${ }^{14,16,30,31}$ While dengue incidence varies by location and year in Sri Lanka, ${ }^{32}$ a primary demographic affected by the disease is children. ${ }^{33,34}$ Colombo district is in the wet zone of the island and reports the highest number of dengue cases every year. ${ }^{34,35}$ This study aims to assess household and hospitalization costs of pediatric dengue illness in this heavily affected urban area in Sri Lanka.

\section{MATERIALS AND METHODS}

Study population and setting. We conducted a prospective costing study in hospitalized pediatric patients at the Lady Ridgeway Hospital for Children (LRHC) located in the city of Colombo. We chose to undertake a prospective study to increase the reliability and validity of the cost data collected through interviews from caregivers. $\mathrm{LRHC}$ is a tertiary care hospital in Colombo district and the National Center of Excellence for Pediatric Care in Sri Lanka. The hospital has 910 beds and provides free-of-charge outpatient and inpatient care for dengue and other childhood illnesses. Pediatric patients (aged 12 years or younger) who were clinically diagnosed with dengue according to a standard dengue case definition based on the 1997-2011 WHO classification ${ }^{36}$ or had laboratory-confirmed dengue ${ }^{37}$ were eligible to participate. Enrollment criteria required that parents or legal guardians accompanying pediatric patients gave consent for children to participate, patients had no comorbidities, and patients had not been partially treated at another hospital. Recruitment took place from August to October in 2013, which 
coincided with peak dengue transmission season after the southwest monsoon that brings heavy rainfall to the western and southern regions of the country every year.

Data collection and management. Household costs. A patient questionnaire was developed for the study (see Supplemental Appendix 1) in English and translated and backtranslated by professional medical translators to the two official local languages, Sinhala and Tamil. The questionnaire was pretested before administration and had three modules with 40 close-ended and 25 open-ended questions. The first two modules were administered by trained interviewers and included questions on demographic and socioeconomic characteristics of patients and their household members; health-seeking behavior before hospitalization at LRHC; characteristics of dengue illness; utilization of healthcare services; household spending and coping strategies; work and school absenteeism; and household income loss due to dengue illness. The third module was self-administered by parents or legal guardians of patients and included 10 openended questions, capturing information on healthcare utilization and associated out-of-pocket spending, work and school absenteeism, and household income loss during the period from hospital discharge to full recovery. These posthospitalization costs were included in total out-of-pocket costs.

Three preintern medical doctors, fluent in English, Sinhala, and Tamil, received a 4-hour training on administration of the questionnaire and were observed during practice interviews. Patients were enrolled from August 22, 2013 to October 15, 2013, and each parent or legal guardian was interviewed face-to-face two or three times during hospital stay. The first interview was held on the first or second day of hospital admission, and the final interview always took place on the day of hospital discharge. The self-administered questionnaire module was provided to all caregivers at hospital discharge with instructions to complete and return it by mail using a prepaid and preaddressed envelope. All data were entered into Microsoft Access Database (2015, Microsoft Corp, Redmond, WA) and analyzed using the statistical software R (version 3.2.2, The R Foundation, Vienna, Austria).

Hospitalization costs. To analyze the direct medical costs of hospitalization for dengue at $\mathrm{RHC}$, the same team of trained preintern medical doctors extracted information on the final clinical diagnosis (DF or DHF/DSS) based on the national dengue diagnosis guidelines, ${ }^{37}$ quantity of medications and medical supplies used for treatment and length of hospital stay from bed-head tickets of patients. These costs are likely generalizable to most other public hospitals. We obtained an average per-patient cost for laboratory tests by disease severity from the hospital database in the absence of more detailed information. Using these data, an average medical cost per episode was calculated by disease severity (DF and DHF/ DSS). We also extracted information on the hospital's operating costs from its annual operating budget to estimate how much it costs to LRHC to run a hospital bed per day, described by $\mathrm{WHO}$ as the hotel cost. ${ }^{38}$ Extracted data on hospital operating costs included nonmedical personnel, communication, electricity, laundry, meals, and administration costs.

Analytical framework and statistical analysis. Household costs. A "hospitalized dengue illness episode" was the unit of analysis in this study. Out-of-pocket costs included direct (medical and nonmedical) and indirect costs (income losses) borne by households due to hospitalized dengue illness. The nature of these costs and the assumptions made in the analysis are described in see Supplemental Appendix 2. The total out-of-pocket cost per episode was obtained by summing direct and indirect costs per illness episode and estimated using two methods to deal with missing data in different cost categories. First, we performed the analysis on a limited part of the dataset: patients with "complete cost data," an approach known as complete-case analysis. ${ }^{39}$ Second, the mean cost in each cost category was estimated by averaging over all "available" data in that category. To ensure unbiased and reliable results, the amount of missing information should be limited. ${ }^{39}$

Hospitalization costs. The direct medical cost of hospitalization per episode was calculated by combining the medical cost of hospitalization (medical staff, medications and medical supplies, and diagnostic tests) with the cost of hospital stay by disease severity. We used the distribution of patients' average length of hospital stay to derive standard deviations in cost estimates. The nature of these costs and the assumptions made in the estimation of medical costs of hospitalization and costs of hospital stay are described in detail in Supplemental Appendix 2. We additionally used Student's $t$-test to determine whether cost differences are statistically significant by disease severity (DF versus DHF/DSS), and we set the significance level at $0.05(P<0.05) .{ }^{40}$

Ethics statement. This study received ethical approval from the institutional review board of the Sri Lankan Ministry of Health, and all data were anonymized before analysis. This research was funded by EU/FP7 through the DengueTools Consortium (grant no. 282589).

\section{RESULTS}

Study population and dengue illness characteristics. A total of 225 children participated in the study. Nine were

TABLE 1

General characteristics of a prospective cohort of hospitalized pediatric dengue patients and their accompanying caregivers at Lady Ridgeway Hospital for Children, Colombo district, Sri Lanka, 2013. Patient sex, $n(\%)$

\begin{tabular}{lr}
\hline Male & $118(55)$ \\
Female & $98(45)$ \\
Patient age (years), $n(\%)$ & $59(27)$ \\
$0-3$ & $63(30)$ \\
$4-7$ & $91(42)$ \\
$8-12$ & $3(1)$ \\
Unknown & \\
Patient currently studying, $n(\%)$ & $154(71)$ \\
Yes & $62(29)$ \\
No & \\
Caregiver's relationship to child, $n(\%)$ & $5(2)$ \\
Father & $188(87)$ \\
Mother & $16(8)$ \\
Grandmother & $7(3)$ \\
Other & \\
Caregiver as primary caregiver, $n(\%)$ & $196(91)$ \\
Yes & $20(9)$ \\
No & \\
Caregiver currently working for pay, $n(\%)$ & $43(20)$ \\
Yes & $172(79)$ \\
No & $1(1)$ \\
Unknown &
\end{tabular}


later dropped from the analysis due to discrepancies in hospitalization dates, leaving a sample size of 216 . The general characteristics of hospitalized dengue patients and their dengue illness episodes are summarized in Tables 1 and 2. Overall, $45 \%$ were female and $39 \%$ were aged 5 years or younger. More than half of patients were ethnically Sinhala (127, $59 \%)$ and religiously Buddhist (118, 55\%), compared with Sri Lankan Moor (53, 25\%) or Sri Lankan Tamil (27, 13\%). Patients in our study who were ethnically Sinhala were slightly underrepresented compared with the population of Sri Lanka (75\%) and Colombo (77\%); otherwise, the sample was representative of the overall population, especially in terms of median income (US\$230 versus US\$241). ${ }^{41}$ The majority of patients $(154,71 \%)$ were attending school. The median number of family members was 4.0 (SD 1.1). Patients were primarily accompanied by their mothers $(188,87 \%)$, and nearly all caregivers (196, $91 \%)$ described themselves as the primary caregiver. Only a minority of accompanying caregivers $(43,20 \%)$ were currently working for pay. Of those working for pay, nearly all $(40,93 \%)$ reported workdays lost with a median of 4 (SD 1.9) days, and more than half $(25,58 \%)$ used paid leave from employer.

Of the 216 hospitalized patients, 170 (79\%) had DF, 45 (20\%) had DHF, and 1 (1\%) had DSS. Twenty-nine (13\%) cases were laboratory confirmed, and 186 (86\%) cases were diagnosed by standard case definition using clinical features and complete blood count values. The average length of hospital stay was 3.1 (SD 1.6) and 4.1 (SD 1.7) days for DF and DHF/DSS patients, respectively. Only one patient with DHF received care in intensive care unit. The mean duration of fever for DF patients was 4.9 (SD 1.6) days and for DHF patients was 5.6 (SD 1.8) days. Nearly all patients (209, 97\%) were reported to have had a fever. Almost all caregivers $(213,99 \%)$ reported other symptoms. The majority of patients (194, 90\%) were reported to have been in very good or excellent health the day before onset of dengue illness.

Utilization of health care services. On average, the majority of caregivers $(178,82 \%)$ reported seeking care from a medical provider within 24 hours of illness onset, and 12\% (26) within 24 to 48 hours of illness onset, while 5\% (11) waited more than 48 hours (Table 3). Of the $30 \%$ (64) of caregivers who did not seek outpatient care before hospitalization, almost all $(62,97 \%)$ sought medical care within 24 hours, whereas only $77 \%$ (116) of caregivers who first sought outpatient care did so within 24 hours. Almost all caregivers (196, $91 \%$ ) used home treatment and remedies first, whereas only $9 \%$ (20) reported seeking professional care immediately. About 70\% (152) of caregivers reported seeking outpatient care prior to hospitalization at LRHC. Of these, $123(81 \%)$ visited a private health facility, $28(18 \%)$ a government health center, and 1 (1\%) an ayurvedic government health center that provide alternative medicine services.
Dengue illness affected school attendance of patients and productive activities of their caregivers and other household members. Of the 154 patients currently attending school, 134 (62\%) missed school for a mean of 4.6 (SD 1.9) days during the period from illness onset to hospital discharge. The mean days of work lost among caregivers was 4.5 (SD 1.9) during this period. All caregivers indicated that household members spent many hours caring for the child post-hospitalization, with a mean of 102 (SD 51.7) hours. The mean number of school days missed for patients was 7.2 (SD 6.7) and of workdays lost for caregivers was 3.6 (SD 3.9) during the period from hospital discharge to full recovery.

Household costs. Table 5 presents the direct (medical and nonmedical), indirect, and total costs of hospitalized pediatric dengue cases to households by disease severity. Out-ofpocket costs in the post-hospitalization period were missing for 111 of the 216 pediatric patients and were excluded from the analysis of household costs. Using Student's $t$-test, we showed that observed characteristics are similar among those with and without missing data (sex, $t=-0.92, P=0.36$ ), (age, $t=-0.51 ; P=0.61$ ), or (religion, $t=-0.09 ; P=0.52$ ). Another five patients were missing some cost data, and these missing costs were imputed.

When we included only the patients with complete cost data $(N=100)$, the mean total cost of a hospitalized dengue episode to a household was estimated at US\$59 (SD 49). The mean direct medical and nonmedical costs were estimated at US\$10 (SD 16) and \$35 (SD 24) per episode, respectively, and the mean indirect cost to the household at US\$15 (SD 29) per episode. Using imputation to infer the missing costs, we computed the mean total cost at US\$58 (SD 50) ( $N=105$ patients). Including all children with complete cost data only from illness onset to hospital discharge $(N=137)$ and hence excluding cost data during post-hospitalization, the mean total cost was marginally lower at US\$57 (SD 60) (Supplemental Appendix 3). Given that all three estimated means were similar, we have a high degree of confidence in the results.

For the complete-case analysis $(N=100)$, the cost differences for DF and DHF/DSS patients were substantial, albeit not statistically significant, across direct nonmedical cost $(t=-1.83 ; P=0.08)$ and indirect cost categories $(t=-1.69 ; P=$ $0.09)$, and statistically significant for the total $(t=-2.02 ; P=$ 0.05 ) cost category. There was no statistically significant difference in direct medical costs between DF and DHF/DSS patients $(t=0.61 ; P=0.55)$. In the available-case analysis, similarly, the differences in direct medical cost were statistically insignificant and the cost differences for DF and DHF/ DSS patients were statistically significant across direct nonmedical $(t=-2.21 ; P=0.03)$ and total $(t=2.34 ; P=0.02)$ cost categories, and insignificant though substantial for the indirect $(t=-1.77 ; P=0.08)$ cost category.

TABLE 2

General characteristics of hospitalized pediatric dengue cases by disease severity at Lady Ridgeway Hospital for Children, Colombo district, Sri Lanka, 2013.

\begin{tabular}{lcc}
\hline & DF & DHF/DSS \\
\hline Length of hospital stay (days), mean (SD) & $3.1(1.6)$ & $4.1(1.7)$ \\
Dengue diagnosis at hospital discharge, $n(\%)$ & $170(79)$ & $4.3(1.7)$ \\
Duration of fever (days), mean (SD) & $4.9(1.6)$ & $216(100)$ \\
\hline $\mathrm{DF}=$ dengue eever; DHF = dengue hemorrhagic fever; DSS = dengue shock syndrome. & $5.1(1.6)$ \\
\hline
\end{tabular}


TABLE 3

Type of health facilities visited and health care providers consulted before hospitalization for pediatric dengue illness at Lady Ridgeway Hospital for Children, Colombo district, Sri Lanka, 2013.

\begin{tabular}{|c|c|c|c|}
\hline & $\mathrm{DF}$ & DHF/DSS & All \\
\hline \multicolumn{4}{|c|}{$\begin{array}{l}\text { Time between onset of illness and seeking } \\
\text { first treatment (hours), } n(\%)\end{array}$} \\
\hline$<24$ & $150(89)$ & $28(61)$ & $178(82)$ \\
\hline $24-48$ & $13(8)$ & $13(28)$ & $26(12)$ \\
\hline$>48$ & $6(4)$ & $5(11)$ & $11(5)$ \\
\hline \multicolumn{4}{|l|}{ First measure of care } \\
\hline Home treatment & $159(94)$ & $37(80)$ & $196(91)$ \\
\hline Professional care & $11(6)$ & $9(20)$ & $20(9)$ \\
\hline \multicolumn{4}{|l|}{ Visit a healer } \\
\hline Yes & $2(1)$ & $0(0)$ & $2(1)$ \\
\hline No & $168(99)$ & $46(100)$ & $214(99)$ \\
\hline \multicolumn{4}{|l|}{ Sought outpatient care first } \\
\hline Yes & $115(68)$ & $37(80)$ & $152(70)$ \\
\hline Western private & $93(81)$ & $30(81)$ & $123(81)$ \\
\hline Western government & 21 (18) & $7(19)$ & $28(18)$ \\
\hline Ayurvedic government & $1(1)$ & $0(0)$ & $1(1)$ \\
\hline No & $55(32)$ & $9(20)$ & $64(30)$ \\
\hline
\end{tabular}

Almost all patients $(205,95 \%)$ were reported to receive freeof-charge medical care at LRHC. Yet nearly all households (202, 94\%) reported using their income or savings to pay for some component of medical care. Only 4\% (8) of households had to borrow money to finance medical care, and only a few households reported selling assets for the same purpose. Only a small proportion of patients $(20,9 \%)$ were covered by health insurance; of those, $85 \%$ (17) of caregivers reported that insurance would reimburse at least some medical costs, and $70 \%$ (14) reported expecting full coverage of costs.

Hospitalization costs. Table 6 presents the direct medical costs of dengue hospitalization by disease severity $(N=216)$. The mean hospitalization cost for DF was estimated at US $\$ 68.0$ (SD 31.4), with the medical and hotel costs of US\$36.5 (SD 16.8) and US\$31.5 (SD 15.8) per episode, respectively. The mean cost of hospitalization for DHF/DSS was estimated at US $\$ 122.7$ (SD 65.2) and was a higher because of higher medical costs totaling to US\$81.3 (SD 41.8) with average medical staff cost of US $\$ 34.5$ (compared with US\$18.4 for DF) and average diagnostic cost of US\$32.5 (compared with US\$17.4 for DF).

\section{DISCUSSION}

As dengue transmission continues to intensify with more frequent and severe epidemics in Sri Lanka and in the broader region of southeast Asia, the disease threatens to become a larger health and economic burden on populations and healthcare systems. In the past 2 decades, the number of dengue cases has increased more than 10-fold in Sri Lanka, and approximatley one-third of cases are reported in children. ${ }^{19}$ The years surrounding 2013 followed generally similar courses of dengue incidence, but since circa 2015, the incidence of dengue has risen. In 2017, during the largest dengue epidemic in Sri Lanka, young children (aged 5-9 years) were reported to be the most affected age group, with an incidence rate of 1,742 per 100,000 population in Western province, which includes Colombo district, followed by young adults. ${ }^{32}$ This study found that the mean out-of-pocket cost of a hospitalized pediatric dengue illness episode was US $\$ 50$ for DF and US\$81 for DHF/DSS. A previous study in the same setting found a lower cost of US\$31 for DF, and US\$37 for DHF. ${ }^{42}$ This is probably because we also included out-ofpocket expenses incurred post-hospitalization until the patient was no longer experiencing symptoms of dengue illness.

Although almost all pediatric patients received free-ofcharge medical care at LRHC, direct medical and nonmedical costs still posed a significant financial burden on households, in addition to indirect costs. Positive, nonzero direct medical costs were primarily due to out-of-pocket payments for outpatient care received at private healthcare facilities before hospitalization. Direct nonmedical costs are not covered by the state-funded healthcare system in Sri Lanka, and our study findings showed that these nonmedical costs are a

TABLE 4

Impact of hospitalized pediatric dengue illness on school attendance and productive activities of households, Colombo district, Sri Lanka, 2013.

\begin{tabular}{|c|c|c|c|}
\hline & DF & DHF/DSS & All \\
\hline \multicolumn{4}{|c|}{$\begin{array}{l}\text { During the period from onset of illness to } \\
\text { hospital discharge, mean (SD) }\end{array}$} \\
\hline Days of school missed & $4.4(1.9)$ & $5.5(2.1)$ & $4.6(1.9)$ \\
\hline Days of work lost & $4.0(1.6)$ & $5.8(2.4)$ & $4.5(1.9)$ \\
\hline Income loss & $19.3(9.9)$ & 47.4 (17.9) & $28.8(19.3)$ \\
\hline \multicolumn{4}{|c|}{$\begin{array}{l}\text { During the period from hospital discharge } \\
\text { to full recovery, mean (SD) }\end{array}$} \\
\hline Days of school missed & $6.7(5.6)$ & $8.6(8.8)$ & $7.2(6.7)$ \\
\hline Days of work lost & $2.2(2.3)$ & $6.3(5.2)$ & $3.6(3.9)$ \\
\hline Income loss & $6.1(13.1)$ & $5.5(10.9)$ & $5.9(12.5)$ \\
\hline
\end{tabular}

$\mathrm{DF}$ = dengue fever; DHF = dengue hemorrhagic fever; $\mathrm{DSS}$ = dengue shock syndrome. 
TABLE 5

Household out-of-pocket costs of hospitalized pediatric dengue illness by disease severity, Colombo district, Sri Lanka, 2013

\begin{tabular}{|c|c|c|c|}
\hline & DF & DHF/DSS & All \\
\hline \multicolumn{4}{|c|}{ Complete case analysis } \\
\hline Sample size & 70 & 30 & 100 \\
\hline \multicolumn{4}{|l|}{ Direct cost per episode, mean (SD) } \\
\hline Medical & $10(17)$ & $11(11)$ & $10(16)$ \\
\hline Nonmedical & 31 (18) & $44(33)$ & $35(24)$ \\
\hline Indirect cost per episode, mean (SD) & $11(23)$ & 24 (39) & $15(29)$ \\
\hline Total cost per episode, mean (SD) & $52(38)$ & $78(67)$ & $59(49)$ \\
\hline \multicolumn{4}{|c|}{ Available case analysis } \\
\hline Sample size & 74 & 31 & 105 \\
\hline \multicolumn{4}{|l|}{ Direct cost per episode, mean (SD) } \\
\hline Medical & $9(17)$ & $13(13)$ & $10(16)$ \\
\hline Nonmedical & $30(18)$ & $44(33)$ & $35(24)$ \\
\hline Indirect cost per episode, mean (SD) & $11(23)$ & $24(38)$ & $14(29)$ \\
\hline Total cost per episode, mean (SD) & $50(37)$ & $81(67)$ & $58(50)$ \\
\hline
\end{tabular}

major contributor to the financial burden dengue imposes on households. Overall, a household's out-of-pocket spending increased with disease severity (DF versus DHF/DSS) and with laboratory rather than clinical diagnoses, although this cost difference does not hold statistical significance (see Supplemental Appendices). This highlighted the importance of early access to diagnosis and treatment not only for better prognosis but also for reduced economic impact.

Although our results are in agreement with the results of other studies in the region, ${ }^{16,43,44}$ the large cost burden incurred by households and hospitals signifies the importance of continued investment into dengue control and prevention and greater and more equitable access to health care in Sri Lanka. Reduced morbidity and mortality is the primary desired outcome from a public health perspective, but the economic burden is an integral component of the disease burden of dengue. Although an important consideration for national and local decision-makers for health priority-setting and resource allocation decisions, the economic burden of dengue is relatively understudied and poorly quantified in Sri Lanka and other endemic countries. Further, based on the recent increase in dengue incidence, we can extrapolate that although average out-of-pocket and hospitalization costs would remain roughly the same, the bottom-line cost incurred by the government would increase as a result of increased patient loads.

The median household income range in our sample was US\$166-291. According to the Household Income and Expenditure Survey 2012-2013, the median monthly household income was US\$241 in Sri Lanka. ${ }^{41}$ Thus, a hospitalized dengue illness episode accounted for $20 \%$ to $35 \%$ of a household's monthly income and imposed an untrivial cost burden, potentially undermining its financial stability. The problem may be further compounded by the fact dengue infections tend to cluster at the household level, affecting more than one household member at a time; a study in Thailand found that an average of 1.4 family members were affected by dengue per episode. ${ }^{44}$

This study found that the direct medical costs of dengue hospitalization ranged between US\$68-123 at LRHC. These results are directly comparable with the results of a previous study conducted in Sri Lanka that also investigated dengue hospitalization costs (US\$80 for DF and US\$191 for DHF in 2013 US dollars) in pediatric patients. ${ }^{42}$ On the other hand, a previous study in Colombo reported higher hospitalization costs, ranging between US\$215 and 293 for pediatric dengue patients (in 2016 US $\$$ ). ${ }^{14}$ Our estimated costs were lower because we estimated a lower hospital stay cost per episode (US\$32 for DF, \$41 for DHF/DSS) based on the operating budget of the hospital. The previous study estimated it as a fixed cost across all pediatric and adult patients (US\$164 in 2016 US dollars) and across three hospitals during an epidemic year with increased disease severity and assuming a longer hospital stay. Additionally, our estimation of hospitalization costs is only about a third of what would have been expected based on the assumption that the full cost of a hospital day is $\sim 3 \%$ of the annual per capita GNI, which corresponds to US\$104.7 per day in the Sri Lankan setting. ${ }^{45}$ There are three potential reasons for this significant gap of about US $\$ 81$ per day; first, only one of our study's 216 patients received medical care in the intensive care unit, the use

TABLE 6

Direct medical costs of dengue hospitalization in pediatric patients by disease severity at Lady Ridgeway Hospital for Children, Colombo district, Sri Lanka, 2013

\begin{tabular}{|c|c|c|c|}
\hline Cost categories & DF & DHF/DSS & All \\
\hline Sample size & 170 & 46 & 216 \\
\hline Medical cost per episode, mean (SD) & $36.5(16.8)$ & $81.3(41.8)$ & $44.3(29.1)$ \\
\hline Medical staff costs, mean (SD) & $18.2(9.1)$ & $34.5(18.4)$ & $21.6(13.4)$ \\
\hline Consultant & $4.6(2.3)$ & $6.0(3.2)$ & $4.9(2.6)$ \\
\hline Senior registrar & $2.1(1.1)$ & $2.9(1.5)$ & $2.3(1.2)$ \\
\hline Registrars & $4.7(2.4)$ & $13.6(7.2)$ & $6.6(5.3)$ \\
\hline Senior house officers & $4.7(2.4)$ & $6.2(3.2)$ & $5.0(2.6)$ \\
\hline Intern house officers & $2.0(1.0)$ & $5.9(3.1)$ & $2.8(2.3)$ \\
\hline IV treatment costs, mean (SD) & $0.9(0.6)$ & $6.4(17.3)$ & $2.1(8.3)$ \\
\hline Fluids & $0.5(0.5)$ & $5.9(17.1)$ & $1.7(8.3)$ \\
\hline Canula and drip set & $0.4(0.2)$ & $0.5(0.3)$ & $0.4(0.2)$ \\
\hline Diagnostics costs, mean (SD) & $17.4(8.7)$ & $32.5(17.3)$ & 20.5 (12.6) \\
\hline Hospital stay cost per episode, mean (SD) & $31.5(15.8)$ & $41.4(22.2)$ & $33.6(17.7)$ \\
\hline Non-medical staff costs, mean (SD) & $27.9(13.9)$ & $36.6(19.6)$ & $29.7(15.7)$ \\
\hline Administrative costs, mean (SD) & $0.5(0.3)$ & $0.7(0.4)$ & $0.5(0.3)$ \\
\hline Operating costs, mean (SD) & $3.1(1.6)$ & $4.1(2.2)$ & $3.3(1.7)$ \\
\hline Direct medical cost per episode, mean (SD) & $68.0(31.4)$ & $122.7(65.2)$ & 77.9 (45.9) \\
\hline
\end{tabular}


of which would have increased average costs per day significantly due to high-cost care using ventilation and emergency drugs. Second, costs might be lower due to LRHC providing care to only pediatric patients rather than adult patients, who have more severe manifestations of the disease. Third, we do not overlook the possibility that because dengue is endemic within Sri Lanka, LRHC is able to make use of economies of scale in its specialized treatment of pediatric dengue; dengue is common enough where treatment has become an optimized and efficient hospital function, and costs have therefore been minimized. Additionally, our costs may be lower because we did not include cost data on groundskeeping, although previous studies indicate that these costs generally constitute $\leq 1 \%$ of total hospital personnel costs. ${ }^{45}$

There were two main limitations to this study. First, it was conducted in a single public hospital in one province of Sri Lanka with a specific focus on clinically and laboratory diagnosed dengue cases in hospitalized pediatric patients. The costs to households and hospitals is, however, expected to vary depending on the type of hospital where care is received and whether the hospital is public or private. To get a more holistic view of dengue hospitalization costs, multiple study locations, including public and private hospitals, should be used. Additionally, the scope of this study was limited to out-of-pocket spending for hospitalized dengue illness among children. Nonhospitalized pediatric dengue illness has a similarly substantial economic impact on households in endemic settings, which is not captured by this study. ${ }^{46}$

Second, similar to other household-level economic impact studies, this study relied on self-reported costs, which are prone to recall bias, primarily due to inaccurate memory. Previous studies have shown that a recall timeframe of 1 year is appropriate for rarely used health care services (e.g., hospitalization), whereas significantly shorter timeframes are recommended for frequently used services (e.g., doctor visits). ${ }^{47}$ To minimize potential recall bias, caregivers were interviewed several times during hospital stay, with the last interview conducted at hospital discharge; roughly more than half of respondents completed the module that elicited out-ofpocket costs incurred by households during the period from post-hospitalization to full recovery. The issue of selfreporting is also important in the investigation of indirect costs associated with an illness episode. The method of asking respondents how many days they lost work has been shown to overestimate productivity losses. ${ }^{47}$ It is a common coping strategy, particularly in low- and middle-income countries, that other household members fill in for a sick person or for a parent caring for a sick child to maintain household productivity. ${ }^{47}$ Additionally, self-reporting often leads to missing data; to address this limitation in our analysis, we estimated costs using different sample bases based on the pattern of missing data. ${ }^{39}$ The multipronged approach we used to analyze out-of-pocket costs, although frequently employed, is an imperfect strategy that rests on the assumption that there is no systemic connection linking patients with missing data. We found no systematic explanation for the missingness within the data, and forgetfulness and losing the return envelope provided for the self-administered module of the questionnaire are the most likely reasons. Despite these limitations, our study has the most comprehensive list of costs for hospitalized pediatric dengue cases in Sri Lanka to date.
Received September 18, 2020. Accepted for publication March 2, 2021.

Published online May 17, 2021.

Note: Supplemental appendices appear at www.ajtmh.org.

Authors' addresses: Enoka Sonali Fernando, Health Office Egodauyana, Ministry of Health, Colombo, Sri Lanka, E-mail: enokasonalifernando@ yahoo.com. Tyler Y. Headley, Department of Political Science, New York University Abu Dhabi, Abu Dhabi, United Arab Emirates, E-mail: tyh255@nyu.edu. Hasitha Tissera, Epidemiology Unit, Ministry of Health, Colombo, Sri Lanka, E-mail: dr_korelege@yahoo.co.uk. Annelies Wilder-Smith, Heidelberg Institute of Global Health, University of Heidelberg, Heidelberg, Germany, Department of Disease Control, London School of Hygiene and Tropical Medicine, Department of Public Health and Clinical Medicine, London, United Kingdom, and Epidemiology and Global Health Unit, Umeå University, Umeå, Sweden, E-mail: wilder-smith@uni-heidelberg.de. Amala De Silva, Department of Economics, University of Colombo, Colombo, Sri Lanka, E-mail: amala@econ.cmb.ac.lk. Yesim Tozan, School of Global Public Health, New York University, New York, NY, E-mail: tozan@nyu.edu.

\section{REFERENCES}

1. Bhatt S, Gething P, Brady O, Messina J, Farlow A, 2013. The global distribution and burden of dengue. Nature 496: 504-507.

2. Redondo-Bravo L, Ruiz-Huerta C, Gomez-Barroso D, SierraMoros MJ, Benito A, Herrador Z, 2019. Imported dengue in Spain: a nationwide analysis with predictive time series analyses. J Travel Med 26: taz072.

3. Halstead S, Wilder-Smith A, 2019. Severe dengue in travellers: pathogenesis, risk and clinical management. J Travel Med 26: taz062.

4. Osman S, Preet R, 2020. Dengue, chikungunya and Zika in GeoSentinel surveillance of international travellers: a literature review from 1995 to 2020. J Travel Med27: taaa222.

5. Buss I, Genton B, D'Acremont V, 2020. Aetiology of fever in returning travellers and migrants: a systematic review and meta-analysis. J Travel Med 27: taaa207.

6. Tozan Y, Headley TY, Sewe MO, Schwartz E, Shemesh T, Cramer JP, Eberhardt KA, Ramharter M, Harrison N, Leder K, 2019. A prospective study on the impact and out-of-pocket costs of dengue illness in international travelers. Am J Trop Med Hyg 100: $1525-1533$.

7. Guzman MG, Harris E, 2015. Dengue. Lancet 385: 453-465.

8. World Health Organization, 2012. Global Strategy for Dengue Prevention and Control 2012-2020. Geneva, Switzerland: WHO.

9. Roth GA, Abate D, Abate KH, Abay SM, Abbafati C, Abbasi N, Abbastabar H, Abd-Allah F, Abdela J, Abdelalim A, 2018. Global, regional, and national age-sex-specific mortality for 282 causes of death in 195 countries and territories, 1980-2017: a systematic analysis for the Global Burden of Disease Study 2017. Lancet 392: 1736-1788.

10. Horstick O, Tozan Y, Wilder-Smith A, 2015. Reviewing dengue: still a neglected tropical disease. PLoS Negl Trop Dis 9: e0003632.

11. Undurraga E, Halasa $Y$, Shepard D, 2013. Use of expansion factors to estimate the burden of dengue in Southeast Asia: a systematic analysis. PLoS Negl Trop Dis 7: e2056.

12. Standish K, Kuan G, Aviles W, Balmaseda A, Harris E, 2010. High dengue case capture rate in four years of a cohort study in Nicaragua compared to national surveillance data. PLoS Negl Trop Dis 4: e633.

13. Beatty $M$ et al., 2010. Best practices in dengue surveillance: a report from the Asia-Pacific and Americas Dengue Prevention Boards. PLoS Negl Trop Dis 4: e890.

14. Thalagala $N$, Tissera $H$, Palihawadana $P$, Amarasinghe $A$, Ambagahawita A, Wilder-Smith A, Shepard D, Tozan Y, 2016. Costs of dengue control activities and hospitalizations in the public health sector during an epidemic year in urban Sri Lanka. PLoS Negl Trop Dis 10: e0004466.

15. Edillo FE, Halasa YA, Largo FM, Erasmo JNV, Amoin NB, Alera MTP, Yoon I-K, Alcantara AC, Shepard DS, 2015. Economic 
cost and burden of dengue in the Philippines. Am J Trop Med Hyg 92: 360-366.

16. Tozan Y, Ratanawong P, Sewe MO, Wilder-Smith A, Kittayapong $P, 2017$. Household costs of hospitalized dengue illness in semi-rural Thailand. PLoS Negl Trop Dis 11: e0005961.

17. Machado AAV, Estevan AO, Sales A, da Silva Brabes KC, Croda J, Negrao FJ, 2014. Direct costs of dengue hospitalization in Brazil: public and private health care systems and use of WHO guidelines. PLoS Neg/ Trop Dis 8: e3104.

18. Shepard D, Undurraga E, Bentacourt-Cravioto M, Guzman M, Halstead S, Harris E, Mudin R, Murray K, Tapia-Conyer R, Gubler D, 2014. Approaches to refining estimates of global burden and economics of dengue. PLoS Negl Trop Dis 8: e3306.

19. Kanakaratne N, Wahala WM, Messer WB, Tissera HA, Shahani A, Abeysinghe N, de-Silva AM, Gunasekera M, 2009. Severe dengue epidemics in Sri Lanka, 2003-2006. Emerg Infect Dis 15: 192-199.

20. Tissera H, Amarasinghe A, Gungseng S, DeSilva A, Yee L, Session O, 2016. Laboratory-enhanced dengue sentinel surveillance in Colombo district, Sri Lanka: 2012-2014. PLoS Negl Trop Dis 10: e0004477.

21. World Health Organization, Department of Control of Neglected Tropical Diseases and TDR, 2009. Dengue: Guidelines for Diagnosis, Treatment, Prevention and Control. Geneva, Switzerland: WHO.

22. Gibbons RV, Vaughn DW, 2002. Dengue: an escalating problem. BMJ 324: 1563.

23. Rigau-Pérez JG, Clark GG, Gubler DJ, Reiter P, Sanders EJ, Vorndam AV, 1998. Dengue and dengue haemorrhagic fever. Lancet 352: 971-977.

24. Messer W, Gubler D, Harris E, Sivananthan K, Silva Ad, 2003. Emergence and global spread of a dengue serotype 3, subtype III virus. Emerg Infect Dis 9: 800-809.

25. Kutsuna S, 2017. Dengue virus type 2 in travelers returning to Japan from Sri Lanka, 2017. Emerg Infect Dis 23: 1931-1933.

26. Tissera D et al., 2010. Dengue surveillance in Colombo, Sri Lanka: baseline seroprevalence among children. Procedia Vaccinol 2: 109-112.

27. Innis $B, 1997$. Antibody responses to dengue virus infection. In: Gubler D, Kuno G, eds. Dengue and Dengue Hemorrhagic Fever. New York, NY: CAB International.

28. Kulatilaka T, Jayakuru W, 1998. Control of dengue/dengue haemorrhagic fever in Sri Lanka. Dengue Bull 22: 53-61.

29. Tissera H, Samaraweera P, Wijesekara N, Jayamanne B, Chulasiri M, Botheju W, 2014. Civil-military cooperation (CIMIC) for an emergency operation against a dengue outbreak in the western province, Sri Lanka. Dengue Bull 38: 64-77.

30. Torres J, Castro J, 2007. The health and economic impact of dengue in Latin America. Cad Saude Publica 23: S23-S31.

31. Torres M, 1997. Impact of an outbreak of dengue fever: a case study from rural Puerto Rico. Hum Organ 56: 19-27.

32. Tissera HA, Jayamanne BD, Raut $R$, Janaki SM, Tozan $Y$, Samaraweera PC, Liyanage P, Ghouse A, Rodrigo C, de Silva AM, 2020. Severe dengue epidemic, Sri Lanka, 2017. Emerg Infect Dis 26: 682.
33. Tam CC, Tissera $H$, de Silva AM, De Silva AD, Margolis HS, Amarasinge A, 2013. Estimates of dengue force of infection in children in Colombo, Sri Lanka. PLoS Negl Trop Dis 7: e2259.

34. Tissera $\mathrm{H}$, et al., 2014. Burden of dengue infection and disease in a pediatric cohort in urban Sri Lanka. Am J Trop Med Hyg 91: 132-137.

35. Epidemiology Unit, Sri Lanka Ministry of Health, 2012. Sri Lanka. Distribution of Notification (H399) Dengue Cases by Month. Colombo, Sri Lanka: Sri Lanka Ministry of Health.

36. Tissera $\mathrm{H}$, Weeraman $\mathrm{J}$, Amarasinghe $\mathrm{A}$, Wijewickrama $\mathrm{A}$, Palihawadana P, Fernando L, 2014. Expediency of dengue illness classification: the Sri Lankan perspective. WHO SouthEast Asia J Public Health 3: 5-7.

37. Sri Lanka Ministry of Health, 2012. Guidelines on Management of Dengue Fever \& Dengue Haemorrhagic Fever In Children and Adolescents. National Guidelines. Colombo, Sri Lanka: Ministry of Health, Sri Lanka Sri Lanka College of Paediatricians.

38. World Health Organization, 2010. CHOosing Interventions that are Cost-Effective project (WHO-CHOICE). Available at: https:// www.who.int/choice/cost-effectiveness/en/. Acccessed March 13, 2020.

39. Little RJ, Rubin DB, 2019. Statistical Analysis with Missing Data, 3rd edition. Hoboken, NJ: John Wiley \& Sons.

40. Greenland S, Senn SJ, Rothman KJ, Carlin JB, Poole C, Goodman $\mathrm{SN}$, Altman DG, 2016. Statistical tests, $P$ values, confidence intervals, and power: a guide to misinterpretations. Eur J Epidemiol 31: 337-350.

41. Department of Census and Statistics, 2015. Household Income Expenditure Survey-2012/13. Colombo, Sri Lanka: Ministry of Policy Planning, Economic Affairs, Child, Youth, and Cultural Affairs.

42. Senanayake $M$, Jayasinghe $S$, Wijesundera $D$, Manamperi $M$, 2014. Economic cost of hospitalized non-fatal paediatric dengue at the Lady Ridgeway Hospital for Children in Sri Lanka. Sri Lanka J Child Health 43: 205-207.

43. Anderson K, Chunsuttiwat S, Nisalak A, Mammen M, Libraty D, Rothman A, Green S, Vaughn D, Ennis F, Endy T, 2007. Burden of symptomatic dengue infection in children at primary school in Thailand. Lancet 369: 1452-1459.

44. Clark D, Mammen M, Nisalak A, Puthimethee V, Endy T, 2005. Economic impact of dengue fever/dengue hemorrhagic fever in Thailand at the family and population levels. Am J Trop Med Hyg 72: 786-791.

45. Shepard DS, Hodgkin D, Anthony YE, 2000. Analysis of Hospital Costs: A Manual for Managers. Geneva, Switzerland: World Health Organization.

46. Huy R, Wichmann O, Beatty M, Ngan C, Duong S, Margolis HS, Vong S, 2009. Cost of dengue and other febrile illnesses to households in rural Cambodia: a prospective communitybased case-control study. BMC Public Health 9: 1-8.

47. Short ME, Goetzel RZ, Pei X, Tabrizi MJ, Ozminkowski RJ, Gibson TB, DeJoy DM, Wilson MG, 2009. How accurate are selfreports? An analysis of self-reported healthcare utilization and absence when compared to administrative data. J Occup Environ Med 51: 786.

48. 2013. Central Bank of Sri Lanka Current Economic Indicators. Available at: http://www.cbsl.gov.lk/htm/english/_cei/er/e_1. asp. Accessed April 19, 2019. 